\title{
Evaluation of oral glucose tolerance test, $\beta$-cell function and adverse obstetric outcomes
}

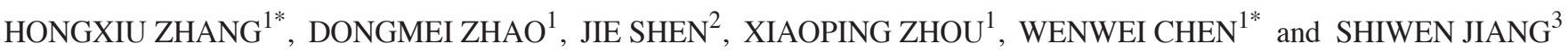 \\ ${ }^{1}$ Department of Obstetrics and Gynecology, The First Affiliated Hospital of Nanjing Medical University, Nanjing, \\ Jiangsu 210036; ${ }^{2}$ HLA Laboratory, The First Affiliated Hospital of Nanjing Medical University, Nanjing, Jiangsu 210029, \\ P.R. China; ${ }^{3}$ Department of Biomedical Sciences, Mercer University School of Medicine, Savannah, GA 31404, USA
}

Received June 28, 2013; Accepted July 12, 2013

DOI: $10.3892 / b r .2013 .136$

\begin{abstract}
This study was conducted in order to investigate the prevalence of impaired glucose tolerance (IGT) and gestational diabetes mellitus (GDM), their effect on pregnancy and their association with adverse obstetric outcomes. A cross-sectional study was performed on 345 pregnant women, who were screened between the 24th and 28th gestational week with a 75 -g oral glucose tolerance test following abnormal results at $1 \mathrm{~h}$ after a $50-\mathrm{g}$ oral glucose challenge test. The obstetric outcomes were recorded along with plasma glucose and insulin levels, homeostasis model assessment of insulin resistance (HOMA-IR) and homeostasis model assessment of $\beta$-cell function index (HOMA- $\beta C F I)$ and 50 subjects were excluded due to incomplete data recovery. Of the 295 pregnant women, $18.6 \%(55 / 295)$ were diagnosed with GDM and 32\% (95/295) with IGT. The GDM group exhibited significantly higher fasting and 1-h blood glucose concentrations compared to the normal glucose tolerance (NGT) and IGT groups $(\mathrm{P}<0.01)$. The 2- and 3-h insulin values of the NGT group were significantly lower compared to those of the GDM group $(\mathrm{P}<0.05$, $\mathrm{P}<0.01)$. In the IGT group, the 2 -h insulin values were higher compared to the NGT group and the 3-h values were significantly higher $(\mathrm{P}<0.01)$, similar to the GDM group. There was a tendency for progressively decreased $\beta$-cell function and increased HOMA-IR from the NGT to the IGT to the GDM group. The adverse outcomes of pregnancy-induced hypertension, fetal distress, neonatal hyperbilirubinemia, preterm delivery, macrosomia and cesarean delivery indicated an association with HOMA- $\beta C F I$ and HOMA-IR. In conclusion,
\end{abstract}

Correspondence to: Dr Wenwei Chen or Dr Hongxiu Zhang, Department of Obstetrics and Gynecology, The First Affiliated Hospital of Nanjing Medical University, 368 North Jiangdong Road, Nanjing, Jiangsu 210036, P.R. China

E-mail: gracechenwenwei@gmail.com

E-mail: hongxiuz@hotmail.com

${ }^{*}$ Contributed equally

Key words: gestational diabetes mellitus, oral glucose tolerance test, insulin resistance pregnancy outcome these findings suggest a clinical significance of $\beta$-cell dysfunction in women with gestational IGT.

\section{Introduction}

Gestational diabetes mellitus (GDM) is associated with an increased risk for adverse obstetrical outcomes, such as macrosomia, shoulder dystocia and birth injury, primary cesarean delivery, preeclampsia, preterm delivery and fetal and neonatal mortality (1-5). Hyperglycemia results from an inadequate compensatory secretion of insulin from the maternal pancreas $(6,7)$. Increased fetal weight in the infants of diabetic mothers was previously considered to be the result of maternal hyperglycemia. However, the control of fetal growth in pregnancies with or without underlying diabetes is significantly more complicated (8).

Asian individuals are classified as genetically highly susceptible to glucose intolerance [i.e., GDM, type 2 diabetes and impaired glucose tolerance (IGT)] (9-12). In the present study, we assessed glucose challenge test (GCT) and 75-g oral glucose tolerance test (OGTT) glucose values, homeostasis model assessment of insulin resistance (HOMA-IR) and several adverse maternal and perinatal outcomes in pregnant Chinese women with a positive GCT. Additionally, we evaluated whether $\beta$-cell function and glucose tolerance status are associated with obstetric outcomes.

\section{Materials and methods}

Subjects. This is a cohort observational study involving prospective enrollment of all consecutive low-risk pregnant women who presented to the antenatal outpatient unit of the First Affiliated Hospital of Nanjing Medical University between April, 2010 and December, 2012. The standard obstetrical practice at our institution includes universal screening for GDM in women between the 24th and 28th week of pregnancy with a 50-g oral GCT. A prenatal 75-g OGTT was subsequently performed with the administration of a standard 75-g glucose load in GCT-positive patients. Exclusion criteria for all subjects included high blood pressure, multiple pregnancies, congenital anomalies or the use of medications known to affect glucose metabolism. Following exclusion, a total of 345 women were recruited. 
This study was approved by the Institutional Review Board of the First Affiliated Hospital of Nanjing Medical University. Informed consent was obtained from all the participants.

GDM screening and glucose tolerance status. Demographic data including age, parity, height, pre-pregnancy weight, pre-pregnancy body mass index (BMI) and family history of diabetes or hypertension were documented for all the subjects. The body weight was stable $( \pm 2 \mathrm{~kg}$ ) for at least 3 months prior to enrollment. The height was measured by a stadiometer and the weight with a digital scale. The BMI was calculated as weight $(\mathrm{kg}) /$ height $^{2}\left(\mathrm{~m}^{2}\right)$. Preceding the diagnostic OGTT, each subject underwent a standard 50-g GCT between the 24th and 28th week of gestation, as part of a universal screening procedure for GDM. Maternal venous blood samples were drawn and plasma was prepared following standard procedures for the measurement of glucose and insulin at fasting and at 1-, 2- and 3-h intervals following glucose administration. Plasma glucose was measured with the glucose oxidase method and insulin concentrations were measured via radioimmunoassay. Based on the results of the OGTT assay according to criteria applying to the Chinese population (13), GDM was diagnosed if two or more glucose values reached or exceeded the following thresholds: fasting, $5.6 \mathrm{mmol} / \mathrm{l} ; 1 \mathrm{~h}, 10.3 \mathrm{mmol} / \mathrm{l} ; 2 \mathrm{~h}, 8.6 \mathrm{mmol} / \mathrm{l}$; and $3 \mathrm{~h}$, $6.7 \mathrm{mmol} / \mathrm{l}$. IGT was diagnosed if one value reached or exceeded these thresholds. The normal glucose tolerance (NGT) group comprised GCT-positive women with normal OGTT values.

Daily glucose profile and management of GDM. Pregnant women diagnosed with GDM were hospitalized in order to undergo dietary management as follows: restriction of carbohydrates to $40 \%$ of the daily calorie intake, which was $25-30 \mathrm{kcal} / \mathrm{kg}$ of ideal body weight for obese and overweight patients and 30-35 kcal/kg for normal-weight subjects. Insulin therapy was initiated when dietary treatment did not consistently maintain fasting and preprandial capillary glucose $\leq 100 \mathrm{mg} / \mathrm{dl}$ and 2 -h postprandial capillary glucose $\leq 120 \mathrm{mg} / \mathrm{dl}$. Regular and neutral protamine Hagedorn (NPH) insulin were used to achieve the glycemic goal and the insulin dose was adjusted according to an insulin algorithm based on bedside glucose monitoring or self-monitoring of capillary glucose values. The patients were discharged after titrating the dosage, with the total insulin dosage at discharge being used for analyzing its correlation with $\beta$-cell function.

Insulin sensitivity and $\beta$-cell function. Insulin sensitivity and insulin secretion were evaluated using measurements from the diagnostic OGTT. The areas under the glucose curve (AUC gluc) and insulin curve (AUC ins) during the OGTT were calculated using the trapezoidal rule. As a measure of insulin secretion, basal insulin and glucose concentrations were used for the estimation of $\beta$-cell secretion according to the homeostasis model assessment (14): HOMA- $\beta$ CFI $=[(20 \mathrm{x}$ insulin $) /($ glucose -3.5$)] \%$; $($ HOMA-IR $)=($ glucose $x$ insulin $) / 22.5$, where the levels of insulin and glucose are expressed in $\mathrm{mU} / \mathrm{l}$ and $\mathrm{mmol} / \mathrm{l}$, respectively (15). The HOMA-IR index was calculated (14) to reflect insulin action in a manner independent of OGTT responses.

Obstetric outcomes. All the pregnant women received routine antenatal examination and the frequency of antenatal visits was increased for those with pregnancy abnormalities. Preterm delivery was defined as any delivery prior to 37 weeks of gestation. Macrosomia was defined as birth weight $\geq 4,000 \mathrm{~g}$. Delivery data and outcomes, including gestational age at delivery, Apgar scores, gender, birth weight, delivery mode, third or fourth degree perineal tears, fetal respiratory distress syndrome, neonatal hyperbilirubinemia and macrosomia, were recorded and analyzed.

Statistical analysis. Data are presented as means \pm standard error of the mean (SEM), unless otherwise noted. One-way analysis of variance (ANOVA) was used to assess the differences of means between groups. Repeated ANOVA variance analysis was used for the comparison of continuous variables between groups. $\mathrm{P}<0.05$ was considered to indicate a statistically significant difference.

\section{Results}

Baseline characteristics of the study groups. From a total of 345 pregnant women who agreed to participate in the study between April, 2010 and December, 2012, complete data from 295 women were obtained for analysis. Fifty women were excluded due to the absence of key data as they had undergone glucose and/or insulin testing or delivery in other hospitals. Of the remaining 295 pregnant women, 55 were diagnosed with GDM and 95 were diagnosed with IGT. Table I summarizes the characteristics of the participants. No significant difference was observed in BMI (pre-delivery and pre-pregnancy BMI), HOMA-IR and homeostasis model assessment of insulin sensitivity (HOMA-IS) among the three groups $(\mathrm{P}>0.05)$. However, the average age of the GDM group was higher compared to that of the IGT and NGT groups $(\mathrm{P}<0.05)$.

Changes of glucose and insulin values and $\beta$-cell function in pregnant women with IGT or GDM. Comparisons of the glucose curve of OGTT among different groups are presented in Fig. 1. There was a statistically significant difference in the IGT vs. the NGT group $(\mathrm{P}<0.001)$ and the GDM vs. the IGT and NGT groups $(\mathrm{P}<0.001)$. Comparisons of the insulin curve of OGTT among different groups are presented in Fig. 2, which reveals that there was no difference in the AUC insulin of OGTT between the groups. The GDM group exhibited significantly higher fasting and 1-h blood glucose levels compared to the NGT and IGT groups $(\mathrm{P}<0.01)$. The 2 -h insulin values of the GDM group were higher compared to those of the NGT and IGT groups $(\mathrm{P}<0.05)$. The 3 -h insulin values of the NGT group were significantly lower compared to those of the IGT and GDM groups $(\mathrm{P}<0.05)$.

Obstetrical outcomes of the study groups. Obstetrical outcomes were compared among the three study groups (Table II). No fourth-degree perineal tears occurred. No shoulder dystocia, perineal tears or postpartum hemorrhage $(\mathrm{PPH})$ were reported among women with diagnosed impaired glucose metabolism. The rate of pregnancy-induced hypertension (PIH), fetal distress, neonatal hyperbilirubinemia, preterm delivery, macrosomia and cesarean delivery exhibited a tendency to increase from the NGT to the IGT to the GDM group (Table II). However, these differences were not statistically significant. 
Table I. Characteristics of the participants.

\begin{tabular}{|c|c|c|c|c|}
\hline Variables & NGT & IGT & GDM & P-value \\
\hline Age (years) & $28.7 \pm 2.2$ & $30.1 \pm 2.7$ & $32.5 \pm 4.5$ & 0.002 \\
\hline Height (m) & $1.60 \pm 0.03$ & $1.62 \pm 0.04$ & $1.61 \pm 0.04$ & 0.482 \\
\hline Pre-pregnancy weight (kg) & $55.71 \pm 7.24$ & $56.29 \pm 10.63$ & $56.00 \pm 7.64$ & 0.973 \\
\hline Pre-pregnancy BMI & $21.5 \pm 2.75$ & $22.6 \pm 3.71$ & $22.0 \pm 3.58$ & 0.986 \\
\hline Pre-delivery weight (kg) & $69.6 \pm 7.0$ & $69.5 \pm 11.4$ & $68.8 \pm 9.9$ & 0.969 \\
\hline Pre-delivery BMI & $27.1 \pm 2.6$ & $26.5 \pm 3.9$ & $26.5 \pm 4.4$ & 0.835 \\
\hline HOMA-IR & $1.73 \pm 0.87$ & $2.09 \pm 1.55$ & $2.27 \pm 0.89$ & 0.334 \\
\hline HOMA- $\beta$ CFI & $191.55 \pm 108.09$ & $174.94 \pm 93.66$ & $147.65 \pm 71.68$ & 0.447 \\
\hline
\end{tabular}

Values are presented as means \pm standard deviation; $\mathrm{P}<0.05$ indicates a statistically significant difference. NGT, normal glucose tolerance; IGT, impaired glucose tolerance; GDM, gestational diabetes mellitus; BMI, body mass index; HOMA-IR, homeostasis model assessment of insulin resistance; HOMA- $\beta$ CFI, homeostasis model assessment of $\beta$-cell function index.

A

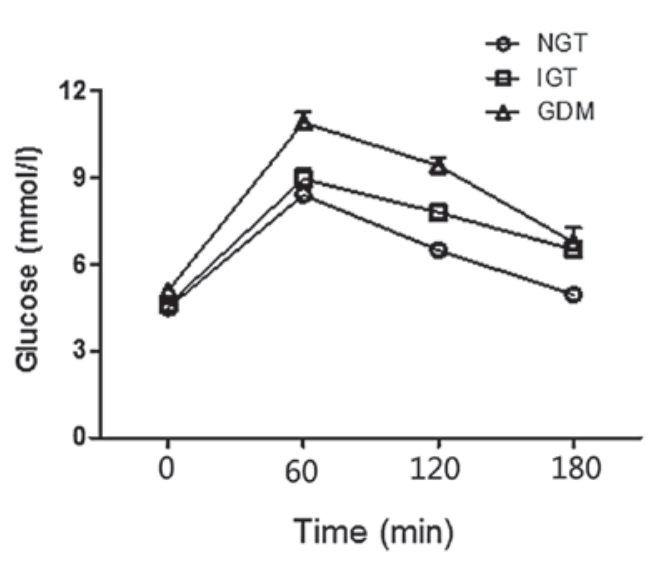

B

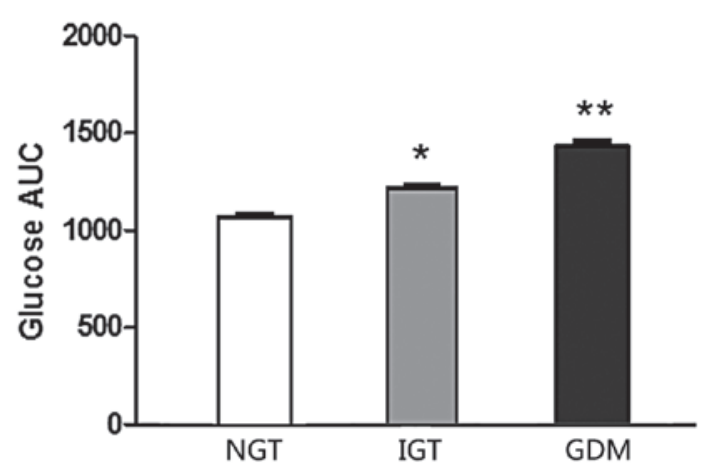

Figure 1. Glucose curve of oral glucose tolerance test (OGTT) in normal glucose tolerance (NGT), impaired glucose tolerance (IGT) and gestational diabetes mellitus (GDM) groups during the 75-g OGTT. There were statistically significant differences in the area under the curve (AUC) of glucose of the OGTT between groups. Data are presented as means \pm standard error of the mean; ${ }^{*}$ IGT vs. NGT, P $<0.001 ;{ }^{* *}$ GDM vs. IGT and NGT, P $<0.001$.

A

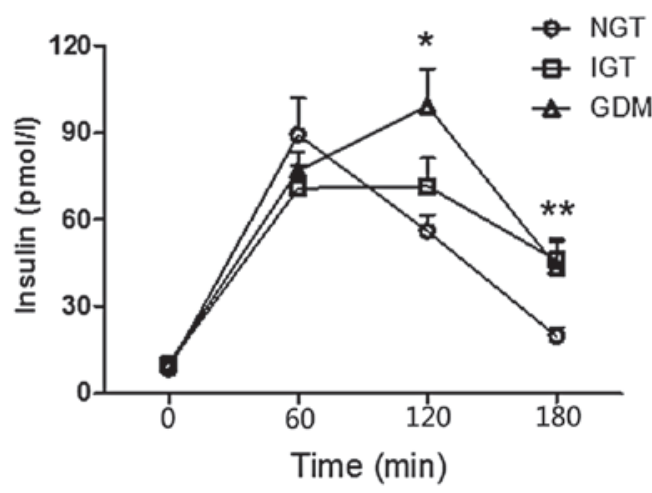

$\mathbf{B}$

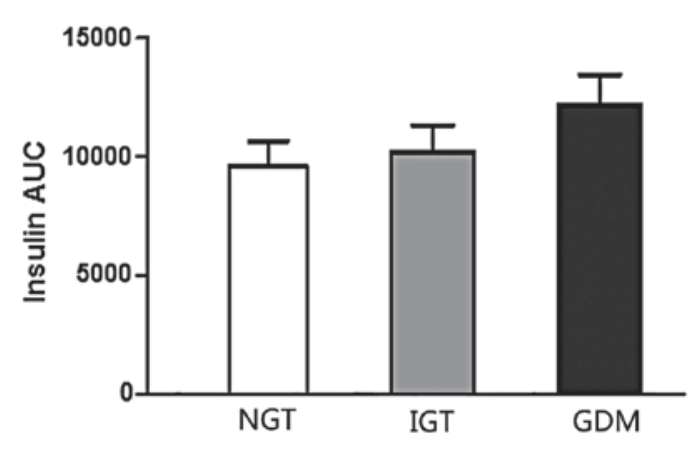

Figure 2. Insulin curve of oral glucose tolerance test (OGTT) in normal glucose tolerance (NGT), impaired glucose tolerance (IGT) and gestational diabetes mellitus (GDM) groups during the 75-g OGTT. There was no difference in the area under the curve (AUC) of insulin of the OGTT between groups. Data are presented as mean \pm standard error of the mean; ${ }^{*}$ NGT vs. GDM, P<0.01; ${ }^{* *}$ NGT vs. IGT and GDM, P<0.01.

\section{Discussion}

The results of our study indicated a tendency for progressively decreased $\beta$-cell function and progressively increased HOMA-IR values from the NGT to the IGT to the GDM group.
Our findings are similar to those previously reported by $\mathrm{Su}$ et al (16). Furthermore, in accordance with the results of previous studies, we also observed an association between increased birth weight and increasing maternal levels of plasma glucose $(17,18)$. 
Table II. Comparison of adverse maternal and neonatal outcomes between NGT, IGT and GDM.

\begin{tabular}{lccc}
\hline Perinatal results & NGT & IGT & GDM \\
\hline PIH (\%) & $10.34 \%(15 / 145)$ & $10.53 \%(10 / 95)$ & $18.18 \%(10 / 55)$ \\
Fetal distress (\%) & $10.34 \%(15 / 145)$ & $21.05 \%(20 / 95)$ & $36.36 \%(20 / 55)$ \\
Neonatal hyperbilirubinemia (\%) & $6.89 \%(10 / 145)$ & $15.79 \%(15 / 95)$ & $18.18 \%(10 / 55)$ \\
Preterm delivery (\%) & $10.34 \%(15 / 145)$ & $10.53 \%(10 / 95)$ & $18.18 \%(10 / 55)$ \\
Birth weight $(\mathrm{kg})$ & $3.335 \pm 0.529$ & $3.476 \pm 0.434$ & $3.613 \pm 0.658$ \\
Macrosomia (\%) & $10.34 \%(15 / 145)$ & $15.79 \%(15 / 95)$ & $18.18 \%(10 / 55)$ \\
Cesarean delivery $(\%)$ & $51.72 \%(75 / 145)$ & $57.89 \%(55 / 95)$ & $81.82 \%(45 / 55)$ \\
\hline
\end{tabular}

NGT, normal glucose tolerance; IGT, impaired glucose tolerance; GDM, gestational diabetes mellitus; PIH, pregnancy-induced hypertension.

Our findings were also similar to those reported by Das et al (15), which demonstrated that the subset of pregnant women who presented with GDM had significantly higher HOMA-IR values and similar HOMA- $\beta C F I$ values compared to pregnant women with NGT. Indeed, high IR is considered a leading risk factor for GDM (19). Our results were in accordance with those of previous studies $(20,21)$ on average HOMA-IS and HOMA-IR values of IGT, indicating that IGT represents an intermediate phenotype between NGT and GDM.

In the present study, a $50-\mathrm{g}$ oral GCT was conducted as part of a standard screening for GDM, as GCT is feasible, easy, user-friendly, cost-effective and convenient for screening purposes (22).

The patients exhibited a delayed insulin peak, which suggested that pregnant women with abnormal glucose metabolism during pregnancy have a decreased IS (Fig. 2). However, there were no differences in IR and IS among the groups. One possible explanation for this observation is that HOMA-IR and HOMA-IS are not the most sensitive evaluation methods. Although HOMA-IR is considered to be a measure of overall IR in pregnancy, it may better reflect liver function rather than peripheral IR (23).

In accordance with the results of a previous study (24), our results demonstrated that women with GDM may be at increased risk for cesarean delivery. The cesarean section rates of the GDM, IGT and NGT groups indicated an inverse association between decreasing cesarean section rates and increasing HOMA- $\beta C F I$ values. The high rate of cesarean section in the GDM group may be due to the fact that women with GDM were at higher risk of birth trauma, PPH and fetal distress, whereas cesarean section was less likely to be considered in NGT. Another reason may be the increased neonatal birth weight in women with GDM. The mean birth weight in the GDM, IGT and NGT groups was $3.613 \pm 0.658,3.476 \pm 0.434$ and $3.335 \pm 0.529 \mathrm{~kg}$, respectively. In our study, the differences among the groups were not statistically significant, similar to previously published data demonstrating that the high glucose levels in the maternal circulation are associated with increased birth weight $(25,26)$. The absence of significant differences in the incidence of adverse obstetric outcomes among the groups may be partly attributed to intervention for GDM, as the results of a previous study which demonstrated that timely intervention for GDM significantly reduced the rate of certain adverse obstetrical outcomes (27).
Fetal growth is a complex process affected by genetics, maternal factors, uterine environment and maternal and fetal hormones. The interference procedures including dietary advice and blood glucose monitoring applied to the GDM group in our study may explain the lack of significant differences in birth weight among the groups. In addition, since the NGT group comprised GCT-positive women with normal OGTT values, there was lack of a GCT-negative screening for GDM to serve as the control group. Women with GDM exhibit a higher risk of subsequent development of type 2 diabetes; therefore, lifestyle modification is encouraged, along with regular screening for diabetes (28).

The exclusion of pregnant women with risk factors affecting glucose metabolism prior to and during pregnancy may represent an advantage of this study, since it reduced the possible interference by factors not directly associated with pregnancy. This prospective study investigated pre-pregnancy and pre-delivery BMI in the GDM, IGT and NGT groups. The use of HOMA-IR is unlikely to lead to erroneous conclusions, since the HOMA-IR value has been found to correlate well with an IS test derived from the insulin-assisted intravenous glucose tolerance test (29).

There were several limitations to the present study that require consideration. This study, by design, was limited to an Asian/Chinese population. The findings may not be extrapolated to non-Asian populations, due to the well-recognized divergence in genetic background and socioeconomic differences. Further studies including other populations are required to investigate whether these findings have a general implication. Furthermore, since the women with NGT in this study exhibited abnormal GCT values, the difference between women with NGT and those with GDM may have been underestimated, since a recent study reported that women without GDM and elevated 50-g GCT values exhibited a higher risk of perinatal morbidity (30).

In conclusion, the prevalence of GDM in our cohort attending a medical centre in China was relatively high (18.6\%). Furthermore, we observed that $\beta$-cell function exhibited a tendency for progressive decrease and HOMA-IR a tendency for progressive increase from the NGT and IGT groups to the GDM group. As regards adverse pregnancy outcomes, the rates of PIH, fetal distress, neonatal hyperbilirubinemia, preterm delivery, macrosomia and cesarean delivery appear to be more closely associated with the glucose and insulin levels 
of OGTT. Further investigations are required to elucidate the association between impaired $\beta$-cell function and its clinical significance in women with GDM.

\section{Acknowledgements}

This study was supported by a grant from the Jiangsu Provincial Department of Education (09kjb320004) and the Jiangsu Provincial Department of Health (JSH-2010-0004).

\section{References}

1. Casey BM, Lucas MJ, McIntire DD and Leveno KJ: Pregnancy outcomes in women with gestational diabetes compared with the general obstetric population. Obstet Gynecol 90: 869-873, 1997.

2. Gokcel A, Bagis T, Killicadag EB, Tarim E and Guvener N: Comparison of the criteria for gestational diabetes mellitus by NDDG and Carpenter and Coustan, and the outcomes of pregnancy. J Endocrinol Invest 25: 357-361, 2002.

3. Yogev Y and Visser GH: Obesity, gestational diabetes and pregnancy outcome. Semin Fetal Neonatal Med 14: 77-84, 2009.

4. Kjos SL and Buchanan TA: Gestational diabetes mellitus. N Engl J Med 341: 1749-1756, 1999.

5. Thompson DM, Dansereau J, Creed M and Ridell L: Tight glucose control results in normal perinatal outcome in 150 patients with gestational diabetes. Obstet Gynecol 83: 362-366, 1994.

6. Homko C, Sivan E, Chen X, Reece EA and Boden G: Insulin secretion during and after pregnancy in patients with gestational diabetes mellitus. J Clin Endocrinol Metab 86: 568-573, 2001.

7. Kautzky-Willer A, Prager R, Waldhausl W, Pacini G, Thomaseth K, Wagner OF, Ulm M, Streli C and Ludvik B Pronounced insulin resistance and inadequate beta-cell secretion characterize lean gestational diabetes during and after pregnancy. Diabetes Care 20: 1717-1723, 1997.

8. Higgins M and Mc Auliffe F: A review of maternal and fetal growth factors in diabetic pregnancy. Curr Diabetes Rev 6: $116-125,2010$.

9. Berkowitz GS, Lapinski RH, Wein R and Lee D: Race/ethnicity and other risk factors for gestational diabetes. Am J Epidemiol 135: 965-973, 1992.

10. Dabelea D, Snell-Bergeon JK, Hartsfield CL, Bischoff KJ, Hamman RF and McDuffie RS: Increasing prevalence of gestational diabetes mellitus (GDM) over time and by birth cohort: Kaiser Permanente of Colorado GDM Screening Program. Diabetes Care 28: 579-584, 2005.

11. Lawrence JM, Contreras R, Chen W and Sacks DA: Trends in the prevalence of preexisting diabetes and gestational diabetes mellitus among a racially/ethnically diverse population of pregnant women, 1999-2005. Diabetes Care 31: 899-904, 2008.

12. Araneta MR, Wingard DL and Barrett-Connor E: Type 2 diabetes and metabolic syndrome in Filipina-American women: a high-risk nonobese population. Diabetes Care 25: 494-499, 2002 .

13. Yue J, Xie X, Lin ZQ, Gou WL and Di W: Gestational diabetes mellitus. In: Obstetrics and Gynecology. Yue J (ed). 7th edition. People's Medical Publishing House, Beijing, pp150-154, 2007.
14. Matthews DR, Hosker JP, Rudenski AS, Naylor BA, Treacher DF and Turner RC: Homeostasis model assessment: insulin resistance and beta-cell function from fasting plasma glucose and insulin concentrations in man. Diabetologia 28: 412-419, 1985.

15. Das S, Behera MK, Misra S and Baliarsihna AK: Beta-cell function and insulin resistance in pregnancy and their relation to fetal development. Metab Syndr Relat Disord 8: 25-32, 2009.

16. Su JB, Wang XQ, Chen JF, Wu G, Jin Y, Xu F, Wang XH and Liu YT: Glycemic variability in gestational diabetes mellitus and its association with $\beta$ cell function. Endocrine 43: 370-375, 2013.

17. HAPO Study Cooperative Research Group, Metzger BE, Lowe LP, Dyer AR, et al: Hyperglycemia and adverse pregnancy outcomes. N Engl J Med 358: 1991-2002, 2008.

18. Sermer M, Naylor CD, Farine D, Kenshole AB, Ritchie JW, Gare DJ, Cohen HR, McArthur K, Holzapfel S and Biringer A: The Toronto Tri-Hospital Gestational Diabetes Project. A preliminary review. Diabetes Care 21 (Suppl 2): B33-B42, 1998.

19. Leinonen A, Hiilesmaa V, Andersen H, Teramo K and Kaaja R: Diurnal blood glucose profiles in women with gestational diabetes with or without hypertension. Diabet Med 21: 1181-1184, 2004.

20. Ergin T, Lembet A, Duran H, Kuscu E, Bagis T, Saygili E and Batioglu S: Does insulin secretion in patients with one abnormal glucose tolerance test value mimic gestational diabetes mellitus? Am J Obstet Gynecol 186: 204-209, 2002.

21. Di Cianni G, Seghieri G, Lencioni C, Cuccuru I, Anichini R, De Bellis A, Ghio A, Tesi F, Volpe L and Del Prato S: Normal glucose tolerance and gestational diabetes mellitus: what is in between? Diabetes Care 30: 1783-1788, 2007.

22. Shrestha A and Chawla CD: The glucose challenge test for screening of gestational diabetes. Kathmandu Univ Med J (KUMJ) 9: 22-25, 2011

23. Kim HS, Chang KH, Yang JI, Yang SC, Lee HJ and Ryu HS: Clinical outcomes of pregnancy with one elevated glucose tolerance test value. Int J Gynaecol Obstet 78: 131-138, 2002.

24. Gorgal R, Goncalves E, Barros M, Namora G, Magalhaes A, Rodrigues $\mathrm{T}$ and Montenegro N: Gestational diabetes mellitus: a risk factor for non-elective cesarean section. J Obstet Gynaecol Res 38: 154-159, 2012.

25. Segregur J, Bukovic D, Milinovic D, Oreskovic S, Pavelic J, Zupic T, Persec J and Pavic M: Fetal macrosomia in pregnant women with gestational diabetes. Coll Antropol 33: 1121-1127, 2009.

26. Lindsay RS, Westgate JA, Beattie J, Pattison NS, Gamble G, Mildenhall LF, Breier BH and Johnstone FD: Inverse changes in fetal insulin-like growth factor (IGF)-1 and IGF binding protein-1 in association with higher birth weight in maternal diabetes. Clin Endocrinol (Oxf) 66: 322-328, 2007.

27. Karakash SD and Einstein FH: Diabetes in pregnancy: glycemia control guidelines and rationale. Curr Opin Endocrinol Diabetes Obes 18: 99-103, 2011.

28. Thanasuan $\mathrm{S}$ and Borriboonhirunsarn D: Incidence of gestational diabetes mellitus among pregnant women with one abnormal value of oral glucose tolerance test. J Med Assoc Thai 89: 1109-1114, 2006.

29. Serlin DC and Lash RW: Diagnosis and management of gestational diabetes mellitus. Am Fam Physician 80: 57-62, 2009.

30. Yee LM, Cheng YW, Liddell J, Block-Kurbisch I and Caughey AB: 50-gram glucose challenge test: is it indicative of outcomes in women without gestational diabetes mellitus? J Matern Fetal Neonatal Med 24: 1102-1106, 2011 